\title{
SOME REMARKS CONCERNING FINITELY SUBADDITIVE OUTER MEASURES WITH APPUCATIONS
}

\author{
JOHN E. KNIGHT \\ Department of Mathematics \\ Long Island University \\ Brooklyn Campus \\ University Plaza \\ Brooklyn, New York 11201 U.S.A.
}

(Received November 21, 1996 and in revised form October 29, 1997)

\begin{abstract}
The present paper is intended as a first step toward the establishment of a general theory of finitely subadditive outer measures. First, a general method for constructing a finitely subadditive outer measure and an associated finitely additive measure on any space is presented. This is followed by a discussion of the theory of inner measures, their construction, and the relationship of their properties to those of an associated finitely subadditive outer measure. In particular, the interconnections between the measurable sets determined by both the outer measure and its associated inner measure are examined. Finally, several applications of the general theory are given, with special attention being paid to various lattice related set functions.
\end{abstract}

KEY WORDS AND PHRASES: Outer measure, inner measure, measurable set, finitely subadditive, superadditive, countably superadditive, submodular, supermodular, regular, approximately regular, condition (M).

1980 AMS SUBJECT CLASSIFICATION CODES: 28A12, 28A10.

\section{INTRODUCTION}

For some time now countably subadditive outer measures have been studied from the vantage point of a general theory (e.g. see [4], [6]), but until now this has not been true for finitely subadditive outer measures. Although some effort has been made to explore the interconnections between certain specific examples of finitely subadditive outer measures (see [3], [7], [8], [10]), there is still no unifying framework for this subject analogous to the one for the countably subadditive case.

This paper is intended to be a first step toward the development of such a general theory for finitely subadditive outer measures. If successful, such an abstract framework would unify the subject, making further research into this area more efficient and thereby enhancing further progress. New examples of finitely subadditive outer measures could be established at will in any space and their characteristics readily inferred from a minimum of information.

Section 3 contains a discussion of the general concept of a finitely subadditive outer measure. Starting from a covering class and an appropriate set function defined on that class, we show how to construct a finitely subadditive outer measure in any space and how this leads to a finitely additive measure in that space.

In Section 4 we explore the general concept of inner measure. Using a suitable finitely subadditive outer measure, we show how to construct an inner measure in any space. We then characterize the measurable sets determined by this inner measure and examine the interconnections between those sets and the ones determined by the original outer measure. The property of submodularity plays a key role in 
this investigation. The results obtained here make it clear that the study of inner measure is essential to a full understanding of finitely subadditive outer measures.

An example of the value of the general principles developed in Sections 3 and 4 is presented in Section 5. We can use a given finite, finitely subadditive outer measure $\nu$ and the collection $\mathcal{S}_{\nu}$ of its measurable sets to define two new finite set functions $\nu^{\circ}$ and $\nu_{0}$. The results of Sections 3 and 4 immediately reveal not only that $\nu^{\circ}$ is a finitely subadditive outer measure and that $\nu_{o}$ is an inner measure, but also their basic properties and their interconnections with one another.

The importance of a general theory of finitely subadditive outer measures begins to emerge in the discussion of the set functions $\nu^{\circ}$ and $\nu_{o}$, for here we first see the marked contrasts between the new theory and that for the countably subadditive case (see [6]). When $\nu^{\circ}$ and $\nu_{0}$ are constructed from a given finite outer measure, certain important related concepts which coincide in the countably subadditive case are actually distinct in the finitely subadditive case. For instance, if $\nu$ is a countably subadditive outer measure and $\mathcal{S}_{\nu}, \mathcal{S}_{\nu}$, and $\mathcal{S}_{\nu_{0}}$ denote the collections of measurable sets for $\nu, \nu^{\circ}$, and $\nu_{o}$, respectively, then $\mathcal{S}_{\nu}=\mathcal{S}_{\nu^{0}}=\mathcal{S}_{\nu_{0}}$. However, if $\nu$ is finitely subadditive, we can only say that $\mathcal{S}_{\nu} \subset \mathcal{S}_{\nu}=\mathcal{S}_{\nu_{0}}$. To obtain complete equality in the latter case, we must impose an additional condition on $\nu$. At the end of Section 5, we see other strong contrasts between the new theory and the old in the concept of regularity.

In Section 6 we apply all the general results of the previous sections to the important examples of measures defined in terms of a lattice, thus obtaining in a systematic manner the fundamental properties of the associated outer and inner measures. (In this connection, see also [3], [7], [8], [10]. For a more detailed discussion of the general theory of finitely subadditive outer measures, see [5]).

In the next section we provide for the reader's convenience a survey of some of the more specialized notation and terminology that we shall use throughout this paper. In the case of lattice related measures and outer measures, our notation and terminology are consistent with [1], [2], [9], [11], [12].

\section{BACKGROUND AND NOTATION}

Throughout this paper, $X$ will denote an arbitrary set and $\mathcal{P}(X)$ the collection of all subsets of $X$. We shall always assume that $X \neq \emptyset$. A collection $\mathcal{L} \subset \mathcal{P}(X)$ will be called a lattice if $A, B \in \mathcal{L}$ implies that $A \cup B \in \mathcal{L}$ and $A \cap B \in \mathcal{L}$. Furthermore, we shall always assume that $\emptyset, X \in \mathcal{L}$. The complement of a set $A \subset X$ will be denoted by $A^{\prime}$, and the collection $\mathcal{L}^{\prime}$ will be defined by $\mathcal{L}^{\prime}=\left\{L^{\prime} \subset X \mid L \in \mathcal{L}\right\}$. Note that $\mathcal{L}^{\prime}$ is a lattice. $\mathcal{A}(\mathcal{L})$ will denote the algebra generated by a lattice $\mathcal{L}$ and $\delta(\mathcal{L})$ the lattice of countable intersections of sets from $\mathcal{L}$. A lattice $\mathcal{L}$ will be called a $\delta$-lattice if $\delta(\mathcal{L})=\mathcal{L}$.

We shall also need the following definition and theorem in Section 6.

DEFINITION 2.1. A lattice $\mathcal{L}$ is said to be normal, if for all $A, B \in \mathcal{L}$ such that $A \cap B=\emptyset$, there exist $C, D \in \mathcal{L}$ such that $A \subset C^{\prime}, B \subset D^{\prime}$, and $C^{\prime} \cap D^{\prime}=\emptyset$.

THEOREM 2.2. A lattice $\mathcal{L}$ is normal if and only if for all $A, L_{1}, L_{2} \in \mathcal{L}$ such that $A \subset L_{1}^{\prime} \cup L_{2}^{\prime}$, there exist $A_{1}, A_{2} \in \mathcal{L}$ such that $A_{1} \subset L_{1}^{\prime}, A_{2} \subset L_{2}^{\prime}$, and $A=A_{1} \cup A_{2}$.

If the property of countable subadditivity is replaced by finite subadditivity in the definition of an outer measure $\nu$, we shall say that $\nu$ is a finitely subadditive outer measure.

We now list some of the less common terminology that we shall use repeatedly throughout this work.

DEFINITION 2.3. An extended real valued set function $\lambda$ defined on a class of sets $\mathcal{E}$ is superadditive on $\mathcal{E}$, if whenever $E, F \in \mathcal{E}, E \cup F \in \mathcal{E}$, and $E \cap F=\emptyset$, then $\lambda(E \cup F) \geq \lambda(E)+\lambda(F)$.

If $\left\{E_{i}\right\}_{i=1}^{n} \subset \mathcal{E}$ is any finite collection of pairwise disjoint sets for which $\bigcup_{i=1}^{n} E_{i} \in \mathcal{E}$ and

$$
\lambda\left(\bigcup_{i=1}^{n} E_{i}\right) \geq \sum_{i=1}^{n} \lambda\left(E_{i}\right),
$$


we shall say that $\lambda$ is finitely superadditive on $\mathcal{E}$. If this same statement holds for any countable collection of pairwise disjoint sets in $\mathcal{E}$ whose union also belongs to $\mathcal{E}$, we say that $\lambda$ is countably superadditive on $\mathcal{E}$.

DEFINITION 2.4. Let $\lambda$ be an extended real valued nonnegative set function defined on a class of sets $\mathcal{E}$. If for all $E, F \in \mathcal{E}$ with $E \cup F \in \mathcal{E}$ and $E \cap F \in \mathcal{E}$, the inequality

$$
\lambda(E \cup F)+\lambda(E \cap F) \leq \lambda(E)+\lambda(F)
$$

holds, we shall say that $\lambda$ is submodular on $\mathcal{E}$. If the reverse inequality holds for any such pair of sets in $\mathcal{E}$, we shall say that $\lambda$ is supermodular on $\mathcal{E}$. If strict equality holds under the same conditions, then $\lambda$ is modular on $\mathcal{E}$. Obviously, a measure defined on an algebra $\mathcal{A}$ is modular on $\mathcal{A}$.

REMARK 2.5. Since submodularity on a class $\mathcal{E}$ implies finite subadditivity on $\mathcal{E}$, we shall refer to a submodular, finitely subadditive outer measure more simply as a submodular outer measure.

We close this section with a brief survey of the measure notation and terminology we shall be using.

For a lattice $\mathcal{L} \subset \mathcal{P}(X)$, we denote by $M(\mathcal{L})$ the collection of all finite, nonnegative, finitely additive, nontrivial measures on $\mathcal{A}(\mathcal{L})$. The subset consisting of all the $0-1$ valued measures in $M(\mathcal{L})$ will be denoted by $I(\mathcal{L})$.

DEFINITION 2.6. (a) A measure $\mu \in M(\mathcal{L})$ is $\mathcal{L}$-regular if for all $A \in \mathcal{A}(\mathcal{L}), \mu(A)=$ $\sup \{\mu(L) \mid A \supset L \in \mathcal{L}\}$.

(b) A measure $\mu \in M(\mathcal{L})$ is $\sigma$-smooth on $\mathcal{L}$, if for all sequences $\left\{L_{n}\right\}_{n=1}^{\infty} \subset \mathcal{L}$ for which $L_{n} \downarrow \emptyset$, then $\mu\left(L_{n}\right) \rightarrow 0$.

(c) A measure $\mu \in M(\mathcal{L})$ is $\sigma$-smooth on $\mathcal{A}(\mathcal{L})$, if for all sequences $\left\{A_{n}\right\}_{n=1}^{\infty} \subset \mathcal{A}(\mathcal{L})$ for which $A_{n} \downarrow \emptyset$, then $\mu\left(A_{n}\right) \rightarrow 0$. (This is equivalent to saying that $\mu$ is countably additive.)

(d) A measure $\mu \in M(\mathcal{L})$ is strongly $\sigma$-smooth on $\mathcal{L}$, if for all sequences $\left\{L_{n}\right\}_{n=1}^{\infty} \subset \mathcal{L}$ for which $L_{n} \downarrow$ and $\bigcap_{n=1}^{\infty} L_{n} \in \mathcal{L}$, then $\mu\left(\bigcap_{n=1}^{\infty} L_{n}\right)=\inf _{n} \mu\left(L_{n}\right)$.

Note: An alternative characterization of this property is: $\mu$ is strongly $\sigma$-smooth on $\mathcal{L}$, if for all sequences $\left\{L_{n}^{\prime}\right\}_{n=1}^{\infty} \subset \mathcal{L}^{\prime}$ for which $L_{n}^{\prime} \uparrow$ and $\bigcup_{n=1}^{\infty} L_{n}^{\prime} \in \mathcal{L}^{\prime}$, then $\mu\left(\bigcup_{n=1}^{\infty} L_{n}^{\prime}\right)=\sup _{n} \mu\left(L_{n}^{\prime}\right)$.

We shall use the following notations to refer to these measures:

$M_{R}(\mathcal{L})$, the subset of all $\mathcal{L}$-regular measures in $M(\mathcal{L})$.

$M_{\sigma}(\mathcal{L})$, the subset of all measures in $M(\mathcal{L})$ which are $\sigma$-smooth on $\mathcal{L}$.

$M^{\sigma}(\mathcal{L})$, the subset of all measures in $M(\mathcal{L})$ which are $\sigma$-smooth on $\mathcal{A}(\mathcal{L})$.

$M_{\sigma *}(\mathcal{L})$, the subset of all measures in $M(\mathcal{L})$ which are strongly $\sigma$-smooth on $\mathcal{L}$.

$M_{R}^{o}(\mathcal{L})$, the subset of all $\mathcal{L}$-regular measures in $M^{\sigma}(\mathcal{L})$.

The corresponding subsets of $I(\mathcal{L})$ are denoted by $I_{R}(\mathcal{L}), I_{\sigma}(\mathcal{L}), I^{\sigma}(\mathcal{L}), I_{\sigma *}(\mathcal{L})$, and $I_{R}^{\sigma}(\mathcal{L})$, respectively.

Note: Clearly, $M^{\sigma}(\mathcal{L}) \subset M_{\sigma *}(\mathcal{L}) \subset M_{\sigma}(\mathcal{L})$.

DEFINITION 2.7. For any lattice $\mathcal{L} \subset \mathcal{P}(X)$ and any measure $\mu \in M(\mathcal{L})$, we define the set function $\mu^{\prime}$ for all $E \subset X$ by

$$
\mu^{\prime}(E)=\inf \left\{\mu\left(L^{\prime}\right) \mid E \subset L^{\prime}, L \in \mathcal{L}\right\} .
$$

DEFINITION 2.8. If $\mathcal{L} \subset \mathcal{P}(X)$ is a lattice and $\mu \in M_{\sigma}(\mathcal{L})$, we define the set function $\mu^{\prime \prime}$ for all $E \subset X$ by

$$
\mu^{\prime \prime}(E)=\inf \left\{\sum_{i=1}^{\infty} \mu\left(L_{i}^{\prime}\right) \mid E \subset \bigcup_{i=1}^{\infty} L_{i}^{\prime}, L_{\imath} \in \mathcal{L}, i=1,2, \cdots\right\} .
$$

DEFINITION 2.9. If $\mathcal{L} \subset \mathcal{P}(X)$ is a lattice, $\mu \in M(\mathcal{L})$, and $\mu^{\prime}$ is the set function of Definition 2.7, then we shall say that $\mu$ is weakly regular if for every $L^{\prime} \in \mathcal{L}^{\prime}$, 


$$
\mu\left(L^{\prime}\right)=\sup \left\{\mu^{\prime}(\tilde{L}) \mid L^{\prime} \supset \tilde{L} \in \mathcal{L}\right\}
$$

We denote the subset of all weakly regular measures in $M(\mathcal{L})$ by $M_{W}(\mathcal{L})$.

Finally, the restriction of a set function $\nu$ to a certain class of $\operatorname{sets} \mathcal{E}$ will be denoted by $\nu \mid \mathcal{E}$.

\section{FINITELY SUBADDITIVE OUTER MEASURES}

In this section we examine the general concept of a finitely subadditive outer measure along with its related properties and its associated finitely additive measures. We begin with

DEFINITION 3.1. An extended real valued set function $\nu$ defined on $\mathcal{P}(X)$ is called a finitely subadditive outer measure on $\mathcal{P}(X)$, if $\nu$ satisfies the following conditions:

(a) $\nu(\emptyset)=0$ and $\nu(E) \geq 0$ for all $E \subset X$.

(b) If $E, F \subset X$ and $E \subset F$, then $\nu(E) \leq \nu(F)$.

(c) For any finite collection $\left\{E_{i}\right\}_{i=1}^{n} \subset \mathcal{P}(X), \nu\left(\bigcup_{i=1}^{n} E_{i}\right) \leq \sum_{i=1}^{n} \nu\left(E_{i}\right)$.

If $\nu(X)<\infty, \nu$ is said to be finite.

DEFINITION 3.2. Let $\nu$ be a finitely subadditive outer measure on $\mathcal{P}(X)$. A set $E \subset X$ is $\nu$-measurable if for every $A \subset X, \nu(A)=\nu(A \cap E)+\nu\left(A \cap E^{\prime}\right)$. The class of all $\nu$-measurable sets will be denoted by $\mathcal{S}_{\nu}$.

Although one can show $\mathcal{S}_{\nu}$ is an algebra and that $\left.\nu\right|_{\nu}$ is a finitely additive measure, these results do not demand that $\nu$ be a finitely subadditive outer measure.

THEOREM 3.3. Let $\lambda$ be a nonnegative real valued set function on $\mathcal{P}(X)$ such that $\lambda(\emptyset)=0$. If

$$
\mathcal{S}_{\lambda}=\left\{E \subset X \mid \forall G \subset X, \lambda(G)=\lambda(G \cap E)+\lambda\left(G \cap E^{\prime}\right)\right\},
$$

then $\mathcal{S}_{\lambda}$ is an algebra and $\bar{\lambda}=\left.\lambda\right|_{\mathcal{S}_{\lambda}}$ is a finitely additive measure.

Theorem 3.3 shows how to construct a finitely additive measure from a finitely subadditive outer measure. The construction of such an outer measure would naturally seem to be the next problem.

DEFINITION 3.4. Let $\mathcal{C} \subset \mathcal{P}(X)$ be nonempty. We say that $\mathcal{C}$ is a covering class if $\emptyset \in \mathcal{C}$ and for every $A \subset X$, there is a finite collection $\left\{E_{i}\right\}_{i=1}^{n} \subset \mathcal{C}$ such that $A \subset \bigcup_{i=1}^{n} E_{i}$.

As in the standard theory of outer measures, we can now construct a finitely subadditive outer measure.

THEOREM 3.5. For any covering class $\mathcal{C} \subset \mathcal{P}(X)$ and any finite, nonnegative set function $\mu$ defined on $\mathcal{C}$ such that $\mu(\emptyset)=0$, the set function $\lambda$ defined for each $A \subset X$ by

$$
\lambda(A)=\inf \left\{\sum_{i=1}^{n} \mu\left(E_{i}\right) \mid A \subset \bigcup_{i=1}^{n} E_{i}, E_{i} \in \mathcal{C}, i=1,2, \cdots, n\right\}
$$

is a finite, finitely subadditive outer measure on $\mathcal{P}(X)$.

By imposing certain conditions on $\mathcal{C}$, we can improve upon Theorem 3.5.

THEOREM 3.6. Let $\mathcal{C}, \mu$, and $\lambda$ be defined as in Theorem 3.5, and suppose $\nu$ is a set function defined for each $A \subset X$ by $\nu(A)=\inf \{\mu(B) \mid A \subset B \in \mathcal{C}\}$.

(a) If $\mathcal{C}$ is closed under finite unions and $\mu$ is finitely subadditive on $\mathcal{C}$, then $\lambda=\nu$. If $\mu$ is monotone, then $\lambda$ extends $\mu$ to a finite, finitely subadditive outer measure on $\mathcal{P}(X)$.

(b) If $\mathcal{C}$ is a lattice and $\mu$ is submodular on $\mathcal{C}$, then $\lambda=\nu$ is a submodular outer measure on $\mathcal{P}(X)$.

PROOF. (a) $\mathcal{C}$ is closed under finite unions, so for every $A \subset X$, there is a $B \in \mathcal{C}$ such that $A \subset B$. Now $A \subset B \cup \emptyset \cup \cdots \cup \emptyset$, so by the definition of $\lambda, \lambda(A) \leq \mu(B)+\mu(\emptyset)+\cdots+\mu(\emptyset)=\mu(B)$. Thus,

$$
\lambda(A) \leq \inf \{\mu(B) \mid A \subset B \in \mathcal{C}\}=\nu(A) .
$$


On the other hand, for each finite collection $\left\{E_{i}\right\}_{i=1}^{n} \subset \mathcal{C}$ for which $A \subset \bigcup_{i=1}^{n} E_{i}, \bigcup_{i=1}^{n} E_{i} \in \mathcal{C}$, so the definition of $\nu$ and the subadditivity of $\mu$ imply that

$$
\nu(A) \leq \inf \left\{\sum_{i=1}^{n} \mu\left(E_{i}\right) \mid A \subset \bigcup_{i=1}^{n} E_{i}, E_{i} \in \mathcal{C}\right\}=\lambda(A) .
$$

It follows from (3.1) and (3.2) that $\lambda=\nu$ on $\mathcal{P}(X)$.

Let $A \in \mathcal{C}$. If $\mu$ is monotone, then

$$
\mu(A) \leq \inf \{\mu(B) \mid A \subset B \in \mathcal{C}\}=\lambda(A),
$$

so $\mu \leq \lambda$ on $\mathcal{C}$. The reverse inequality is clear, so $\lambda=\mu$ on $\mathcal{C}$.

(b) Suppose $A_{1}, A_{2} \subset X$ and let $\epsilon>0$ be given. $\mathcal{C}$ is closed under finite unions, so there exist $\mathrm{B}_{\imath} \in \mathcal{C}$ such that $A_{\imath} \subset B_{i}, i=1,2$, and we may choose the $B_{\imath}$ so that $\mu\left(B_{i}\right)<\nu\left(A_{i}\right)+\epsilon / 2, i=1,2$. Furthermore, $B_{1} \cup B_{2}, B_{1} \cap B_{2} \in \mathcal{C}$, so the definition of $\nu$ and the submodularity of $\mu$ on $C$ imply that

$$
\begin{aligned}
\nu\left(A_{1} \cup A_{2}\right)+\nu\left(A_{1} \cap A_{2}\right) & \leq \mu\left(B_{1} \cup B_{2}\right)+\mu\left(B_{1} \cap B_{2}\right) \\
& \leq \mu\left(B_{1}\right)+\mu\left(B_{2}\right) \\
& <\nu\left(A_{1}\right)+\nu\left(A_{2}\right)+\epsilon .
\end{aligned}
$$

Thus, $\nu$ is submodular. Since part (a) implies $\lambda=\nu$, the desired conclusion follows.

REMARK. Let $\mu_{1}$ and $\mu_{2}$ be finite, nonnegative set functions, each defined on a covering class $\mathcal{C} \subset \mathcal{P}(X)$, vanishing at $\emptyset$, and yielding, according to Theorem 3.5 , finitely subadditive outer measures $\lambda_{1}$ and $\lambda_{2}$, respectively. If $\lambda_{1}=\lambda_{2}$ on $\mathcal{C}$, then $\lambda_{1}=\lambda_{2}$ on $\mathcal{P}(X)$.

Since the concept of regularity for finitely subadditive outer measures will be important in later sections of this paper, we list here for convenience the following theorems and definitions.

DEFINITION 3.7. If $\nu$ is a finitely subadditive outer measure, $A \subset X$, and $E \in \mathcal{S}_{\nu}$, we shall say that $E$ is a measurable cover for $A$ if $A \subset E$ and $\bar{\nu}(E)=\nu(A)$, where $\bar{\nu}=\left.\nu\right|_{S_{\nu}}$. If there is a measurable cover for every $A \subset X$, we shall say that $\nu$ is regular.

THEOREM 3.8. If $\nu$ is a finite, regular, finitely subadditive outer measure, then $E \in \mathcal{S}_{\nu}$ if and only if $\nu(X)=\nu(E)+\nu\left(E^{\prime}\right)$ (see [6]).

With the machinery we have now set up, we can easily construct finitely subadditive outer measures and their associated finitely additive measures in any space. We specify $\mathcal{C}$ and $\mu$ and the finitely subadditive outer measure and its associated finitely additive measure are automatically defined. To determine the properties of these set functions, we need only examine the properties of $\mathcal{C}$ and $\mu$. However, we can learn substantially more about finitely subadditive outer measures in general by examining the related concept of inner measure.

\section{INNER MEASURES}

We turn now to a discussion of the general notion of an inner measure defined on $\mathcal{P}(X)$ and the relationship of its properties to those of a finitely subadditive outer measure.

DEFINITION 4.1. An extended real valued set function $\rho$ defined on $\mathcal{P}(X)$ is an inner measure if it satisfies the following properties:

(a) $\rho(\emptyset)=0$.

(b) For all $E \subset X, \rho(E) \geq 0$.

(c) $\rho$ is monotone.

(d) $\rho$ is countably superadditive on $\mathcal{P}(X)$.

We may state the problem of interest as follows: if we use a finite, finitely subadditive outer measure $\nu$ on $\mathcal{P}(X)$ to define a new set function $\rho$ on $\mathcal{P}(X)$ by $\rho(E)=\nu(X)-\nu\left(E^{\prime}\right)$ for all $E \subset X$, then when is $\rho$ an inner measure? Under the stated conditions, $\rho$ will possess the first three properties of an inner 
measure, but to assure countable superadditivity, $\nu$ must possess additional properties, as the next theorem shows.

THEOREM 4.2. If $\nu$ is a finite, finitely subadditive outer measure, and $\rho$ is defined on $\mathcal{P}(X)$ by $\rho(E)=\nu(X)-\nu\left(E^{\prime}\right)$ for all $E \subset X$, then:
(a) $\rho(\emptyset)=0$.
(b) For all $E \subset X, 0 \leq \rho(E)<\infty$.
(c) $\rho$ is monotone.
(d) $\rho \leq \nu$ on $\mathcal{P}(X)$.
(e) If $E \in \mathcal{S}_{\nu}$, then $\rho(E)=\nu(E)$.
(f) If $\nu$ is submodular, then $\rho$ is a supermodular inner measure.

PROOF. The proofs of parts (a) through (e) are not difficult, so for the sake of brevity, we omit them here and prove only part $(f)$.

$\nu$ is submodular, so if $E, F \subset X$, then

$$
\nu\left(E^{\prime} \cup F^{\prime}\right)+\nu\left(E^{\prime} \cap F^{\prime}\right) \leq \nu\left(E^{\prime}\right)+\nu\left(F^{\prime}\right) .
$$

By the definition of $\rho$ and the finiteness of $\rho$ and $\nu$, statement (4.1) implies

$$
\nu(X)-\rho(E \cap F)+\nu(X)-\rho(E \cup F) \leq \nu(X)-\rho(E)+\nu(X)-\rho(F) .
$$

Hence, $\rho(E \cup F)+\rho(E \cap F) \geq \rho(E)+\rho(F)$, so $\rho$ is supermodular.

If $E \cap F=\emptyset$, then $\rho$ is superadditive on disjoint sets and by induction $\rho$ is finitely superadditive also. Now let $\left\{E_{z}\right\}^{\infty}=1 \subset \mathcal{P}(X)$ be a countable collection of pairwise disjoint sets. Since $\rho$ is monotone, then for all $n$,

$$
\rho\left(\bigcup_{i=1}^{\infty} E_{i}\right) \geq \rho\left(\bigcup_{i=1}^{n} E_{i}\right) \geq \sum_{i=1}^{n} \rho\left(E_{i}\right)
$$

Letting $n \rightarrow \infty$, we have

$$
\rho\left(\bigcup_{i=1}^{\infty} E_{i}\right) \geq \lim _{n \rightarrow \infty} \sum_{i=1}^{n} \rho\left(E_{i}\right)=\sum_{i=1}^{\infty} \rho\left(E_{i}\right) .
$$

Therefore, $\rho$ is an inner measure.

REMARK 4.3. When $\nu$ is a finite, submodular outer measure, the set function $\rho$ defined in Theorem 4.2 will be called the inner measure determined by $\nu$.

We note also that by an argument similar to the one used to show that $\rho$ is supermodular when $\nu$ is submodular in Theorem 4.2(f), we can also show that $\nu$ is submodular whenever $\rho$ is supermodular.

DEFINITION 4.4. If $\lambda$ is an inner measure on $\mathcal{P}(X)$, we shall say that a set $E \subset X$ is $\lambda$-measurable if for every $A \subset X$, we have

$$
\lambda(A)=\lambda(A \cap E)+\lambda\left(A \cap E^{\prime}\right) .
$$

We denote the class of all $\lambda$-measurable sets in $\mathcal{P}(X)$ by $\mathcal{S}_{\lambda}$.

The next theorem follows immediately from Theorem 3.3.

THEOREM 4.5. If $\lambda$ is a finite inner measure on $\mathcal{P}(X)$, then $\mathcal{S}_{\lambda}$ is an algebra and $\bar{\lambda}=\left.\lambda\right|_{\mathcal{\lambda}}$ is a finitely additive measure on $\mathcal{S}_{\lambda}$.

We now have the important

THEOREM 4.6. If $\nu$ is a finite, submodular outer measure and $\rho$ is the inner measure determined by $\nu$, then $\mathcal{S}_{\rho}=\{E \subset X \mid \rho(E)=\nu(E)\}$.

PROOF. Let $\mathcal{S}=\{E \subset X \mid \rho(E)=\nu(E)\}$ and choose $E \in \mathcal{S}_{\rho}$. Letting $A=X$ in Definition 4.4, we have $\rho(X)=\rho(E)+\rho\left(E^{\prime}\right)$. Since $X \in \mathcal{S}_{\nu}$, Theorem 4.2(e) implies that $\rho(X)=\nu(X)$, and by the definition of $\rho, \rho\left(E^{\prime}\right)=\nu(X)-\nu(E)$. Since all quantities are finite, 


$$
\rho(E)=\rho(X)-\rho\left(E^{\prime}\right)=\nu(X)-(\nu(X)-\nu(E))=\nu(E) .
$$

Thus, $E \in \mathcal{S}$, so $\mathcal{S}_{\rho} \subset \mathcal{S}$.

To show the converse, let $E \in \mathcal{S}$. Clearly $\rho\left(E^{\prime}\right)=\nu(X)-\nu(E)=\rho(X)-\rho(E)$, so by the definition of $\rho$,

$$
\rho(X)=\rho(E)+\rho\left(E^{\prime}\right) .
$$

Since $\rho$ is supermodular, then for any $A \subset X$,

$$
\begin{aligned}
& \rho(A \cap E)+\rho(A \cup E) \geq \rho(A)+\rho(E), \\
& \rho\left(A \cap E^{\prime}\right)+\rho\left(A \cup E^{\prime}\right) \geq \rho(A)+\rho\left(E^{\prime}\right) .
\end{aligned}
$$

Adding and applying (4.2) gives

$$
\rho(A \cap E)+\rho\left(A \cap E^{\prime}\right)+\rho(A \cup E)+\rho\left(A \cup E^{\prime}\right) \geq 2 \rho(A)+\rho(X) .
$$

Again, because $\rho$ is supermodular, we obtain

$$
\begin{aligned}
\rho(A \cup E)+\rho\left(A \cup E^{\prime}\right) & \leq \rho\left([A \cup E] \cup\left[A \cup E^{\prime}\right]\right)+\rho\left([A \cup E] \cap\left[A \cup E^{\prime}\right]\right) \\
& =\rho(X)+\rho(A) .
\end{aligned}
$$

Now statements (4.4) and (4.5) imply

$$
\rho(A \cap E)+\rho\left(A \cap E^{\prime}\right)+\rho(X)+\rho(A) \geq 2 \rho(A)+\rho(X) .
$$

Thus, for all $A \subset X, \rho(A \cap E)+\rho\left(A \cap E^{\prime}\right) \geq \rho(A)$. Combining this with the fact that $\rho$ is superadditive, we see that for all $A \subset X$,

$$
\rho(A)=\rho(A \cap E)+\rho\left(A \cap E^{\prime}\right) .
$$

Hence, $E \in \mathcal{S}_{\rho}$, and, therefore, $\mathcal{S}_{\rho} \subset \mathcal{S}$. Combining this result with the reverse inclusion shown above, we have $\mathcal{S}_{\rho}=\mathcal{S}$.

COROLLARY 4.7. Under the hypotheses of Theorem 4.6, $\mathcal{S}_{\nu} \subset \mathcal{S}_{\rho}$.

PROOF. Immediate by Theorems 4.2 and 4.6 .

DEFINITION 4.8. If a finite, finitely subadditive outer measure $\nu$ satisfies the condition that $E \in \mathcal{S}_{\nu}$ if and only if $\nu(X)=\nu(E)+\nu\left(E^{\prime}\right)$, we shall say that $\nu$ satisfies condition (M).

From this we obtain directly the important

THEOREM 4.9. If $\nu$ is a finite, submodular outer measure on $\mathcal{P}(X)$, and $\rho$ is the finite inner measure determined by $\nu$, then $\nu$ satisfies condition (M) if and only if $\mathcal{S}_{\nu}=\mathcal{S}_{\rho}=\{E \subset X \mid \rho(E)=\nu(E)\}$.

PROOF. Since by Theorem 4.6 and Corollary 4.7 we always have $\mathcal{S}_{\nu} \subset \mathcal{S}_{\rho}$, we need only show the conclusion holds for $\mathcal{S}_{\rho} \subset \mathcal{S}_{\nu}$.

Assume that $\nu$ satisfies condition $(M)$ and let $E \in \mathcal{S}_{\rho}$. By the definition of $\rho$, we have $\nu(E)=\rho(E)=\nu(X)-\nu\left(E^{\prime}\right)$, and since $\nu$ is finite, we obtain $\nu(X)=\nu(E)+\nu\left(E^{\prime}\right)$. The hypothesis now implies that $E \in \mathcal{S}_{\nu}$, so that $\mathcal{S}_{\rho} \subset \mathcal{S}_{\nu}$.

On the other hand, suppose $\mathcal{S}_{\rho} \subset \mathcal{S}_{\nu}$. Clearly, if $E \in \mathcal{S}_{\nu}$, then $\nu(X)=\nu(E)+\nu\left(E^{\prime}\right)$, so it will suffice to prove the converse implication. Assuming this latter equality holds for any $E \subset X$, the finiteness of $\nu$ and the definition of $\rho$ imply that $\rho(E)=\nu(X)-\nu\left(E^{\prime}\right)=\nu(E)$. Hence, $E \in \mathcal{S}_{\rho}$, so by hypothesis $E \in \mathcal{S}_{\nu}$, and consequently $\nu$ satisfies condition (M).

It now seems natural to inquire as to when a finitely subadditive outer measure is submodular. One condition that ensures this is given by

THEOREM 4.10. If $\nu$ is a finite, regular, finitely subadditive outer measure, then $\nu$ is submodular.

PROOF. Clear.

We conclude this section with a direct result of this theorem and Theorem 4.2. 
COROLLARY 4.11. Let $\nu$ be a finite, finitely subadditive outer measure and $\rho$ the set function defined for all $E \subset X$ by $\rho(E)=\nu(X)-\nu\left(E^{\prime}\right)$. If $\nu$ is regular, then $\rho$ is an inner measure.

\section{AN APPLICATION TO THE SET FUNCTIONS $\nu^{\circ}$ AND $\nu_{0}$}

Beginning with a finitely subadditive outer measure $\nu$ and the collection $S_{\nu}$ of $\nu$-measurable sets, we can define two new set functions $\nu^{\circ}$ and $\nu_{o}$ on $\mathcal{P}(X)$. We shall then show how to derive their basic properties using the general theory of Sections 3 and 4 . We then follow this with a full discussion of the interconnections between the properties of $\nu^{\circ}$ and $\nu_{0}$ and also of their relationship to the original outer measure $\nu$.

Throughout this section, $\nu$ will always denote a finite, finitely subadditive outer measure on $\mathcal{P}(X)$ and $\mathcal{S}_{\nu}$, the set of all $\nu$-measurable sets.

DEFINITION 5.1. For all $E \subset X$, we define the set functions $\nu^{\circ}$ and $\nu_{o}$ as follows:

$$
\begin{aligned}
& \nu^{\circ}(E)=\inf \left\{\nu(M) \mid E \subset M \in \mathcal{S}_{\nu}\right\} \\
& \nu_{o}(E)=\sup \left\{\nu(M) \mid E \supset M \in \mathcal{S}_{\nu}\right\}
\end{aligned}
$$

THEOREM 5.2. (a) For every $E \subset X, \nu_{0}(E) \leq \nu(E) \leq \nu^{\circ}(E)$.

(b) $\nu^{\circ}$ is a finite, submodular outer measure on $\mathcal{P}(X)$, and if $E \in \mathcal{S}_{\nu}$, then $\nu^{\circ}(E)=\nu(E)$.

(c) $\nu_{o}$ is a finite, supermodular inner measure on $\mathcal{P}(X)$. For all $E \subset X$,

$$
\nu_{o}(E)=\nu(X)-\nu^{\circ}\left(E^{\prime}\right)
$$

PROOF. (a) Clear.

(b) $\mathcal{S}_{\nu}$ is a lattice and $\bar{\nu}=\left.\nu\right|_{\nu}$ is submodular on $\mathcal{S}_{\nu}$, so by Theorem 3.6(b), $\nu^{\circ}$ is a submodular outer measure on $\mathcal{P}(X)$. Since by Theorem 3.6(a), $\nu^{\circ} \mid S_{\nu}=\nu$, then $\nu^{\circ}(E)=\nu(E)$ for any $E \in \mathcal{S}_{\nu}$. Clearly, $\nu^{\circ}$ is finite.

(c) Since $\bar{\nu}=\left.\nu\right|_{\nu}$ is a finitely additive measure on $\mathcal{S}_{\nu}$, then for $E \subset X$, Definition 5.1 implies

$$
\begin{aligned}
\nu_{o}(E) & =\sup \left\{\bar{\nu}(M) \mid E \supset M \in \mathcal{S}_{\nu}\right\} \\
& =\sup \left\{\bar{\nu}(X)-\bar{\nu}\left(M^{\prime}\right) \mid E^{\prime} \subset M^{\prime} \in \mathcal{S}_{\nu}\right\} \\
& =\bar{\nu}(X)-\inf \left\{\bar{\nu}\left(M^{\prime}\right) \mid E^{\prime} \subset M^{\prime} \in \mathcal{S}_{\nu}\right\} \\
& =\nu(X)-\nu^{\circ}\left(E^{\prime}\right) .
\end{aligned}
$$

Part (b) above and Theorem 4.2(f) now imply that $\nu_{o}$ is a supermodular inner measure. Clearly, $\nu_{o}$ is also finite.

REMARK 5.3. We define a $\nu^{\circ}$-measurable set according to Definition 3.2 and denote the collection of all these sets by $\mathcal{S}_{\nu}$. By Theorem 3.3, $\mathcal{S}_{\nu}$ is an algebra and $\bar{\nu}^{\circ}=\left.\nu^{\circ}\right|_{\mathcal{v}}$ is a finitely additive measure. Similarly, we define a $\nu_{0}$-measurable set according to Definition 4.4 and denote the class of all such sets by $\mathcal{S}_{\nu_{0}}$. This leads directly to

THEOREM 5.4. (a) $\mathcal{S}_{\nu_{0}}$ is an algebra and $\bar{\nu}_{0}=\nu_{0} \mid S_{\nu_{0}}$ is a finitely additive measure on $\mathcal{S}_{\nu_{0}}$.

(b) $E \in \mathcal{S}_{\nu_{o}}$ if and only if $\nu_{o}(A) \leq \nu_{o}(A \cap E)+\nu_{o}\left(A \cap E^{\prime}\right)$, for all $A \subset X$.

(c) $\mathcal{S}_{\nu_{o}}=\left\{E \subset X \mid \nu_{o}(E)=\nu^{\circ}(E)\right\}$.

(d) $\mathcal{S}_{\nu} \subset \mathcal{S}_{\nu^{0}} \subset \mathcal{S}_{\nu_{0}}$.

(e) If $E \in \mathcal{S}_{\nu}$ (or $\mathcal{S}_{\nu}$ ), then $\nu_{o}(E)=\nu(E)=\nu^{\circ}(E)$.

(f) $\nu^{\circ}$ satisfies condition (M) if and only if $\mathcal{S}_{\nu^{\circ}}=\mathcal{S}_{\nu_{0}}$.

PROOF. (a) Clear, by Theorem 4.5.

(b) This follows by the superadditivity of $\nu_{0}$.

(c) An immediate consequence of Theorems 4.6 and $5.2(b, c)$.

(d) Let $E \in \mathcal{S}_{\nu}$ and choose any $A \subset X$. Given $\epsilon>0$, there exists an $M \in \mathcal{S}_{\nu}$ such that $A \subset M$ and $\nu(M)<\nu^{\circ}(A)+\epsilon$. Since $A \cap E \subset M \cap E \in \mathcal{S}_{\nu}$ and $A \cap E^{\prime} \subset M \cap E^{\prime} \in \mathcal{S}_{\nu}$, the definition of $\nu^{\circ}$ implies 


$$
\begin{aligned}
\nu^{\circ}(A)+\epsilon>\nu(M) & =\nu(M \cap E)+\nu\left(M \cap E^{\prime}\right) \\
& \geq \nu^{\circ}(A \cap E)+\nu^{\circ}\left(A \cap E^{\prime}\right),
\end{aligned}
$$

and therefore $\nu^{\circ}(A) \geq \nu^{\circ}(A \cap E)+\nu^{\circ}\left(A \cap E^{\prime}\right)$. Hence $E \in \mathcal{S}_{\nu}$, and thus $\mathcal{S}_{\nu} \subset \mathcal{S}_{\nu \text {. }}$ Corollary 47 gives $\mathcal{S}_{\nu} \subset \mathcal{S}_{\nu_{0}}$.

(e) A clear consequence of parts (c) and (d) above and Theorem 5.2(a).

(f) Follows from Theorems 4.9 and 5.2(b,c).

The following inequalities are not difficult to show and are frequently useful.

LEMMA 5.5. If $E$ and $F$ are disjoint sets in $\mathcal{P}(X)$, then

$$
\nu_{o}(E)+\nu_{o}(F) \leq \nu_{o}(E \cup F) \leq \nu_{o}(E)+\nu^{\circ}(F) \leq \nu^{\circ}(E \cup F) \leq \nu^{\circ}(E)+\nu^{\circ}(F) .
$$

With this lemma we can show

THEOREM 5.6. (a) If $E \in \mathcal{S}_{\nu}$, then for all $A \subset X, \nu_{o}(A \cap E)+\nu^{\circ}\left(A^{\prime} \cap E\right)=\nu(E)$.

(b) If $G \subset E \in S_{\nu}$, then $\nu_{o}(G)=\nu(E)-\nu^{\circ}(E-G)$.

The following important characterization theorem reveals the relationships between $\mathcal{S}_{\nu}, \mathcal{S}_{\nu}$, and $\mathcal{S}_{\nu_{0}}$ when $\nu$ satisfies condition (M).

THEOREM 5.7. If $\nu$ satisfies condition (M), then:

(a) For any $E \subset X$, if $\nu_{o}(E)=\nu(E)$, then $E \in \mathcal{S}_{\nu}$.

(b) $E \in \mathcal{S}_{\nu}$ if and only if $\nu_{o}(E)=\nu^{\circ}(E)$.

(c) $\mathcal{S}_{\nu}=\mathcal{S}_{\nu^{0}}=\mathcal{S}_{\nu_{0}}$.

PROOF. (a) Suppose $\nu_{o}(E)=\nu(E)$ for some $E \subset X$. By Theorem 5.2(a,c),

$$
\nu(X)=\nu(E)-\nu^{\circ}\left(E^{\prime}\right) \geq \nu(E)+\nu\left(E^{\prime}\right) .
$$

But $\nu$ is subadditive, so $\nu(X)=\nu(E)+\nu\left(E^{\prime}\right)$, and since $\nu$ satisfies condition (M), $E \in \mathcal{S}_{\nu}$.

(b) Clear.

(c) By Theorem 5.4(c), if $E \in \mathcal{S}_{\nu_{0}}$, then $\nu_{o}(E)=\nu^{\circ}(E)$. By part (b) above, $E \in \mathcal{S}_{\nu}$ so $\mathcal{S}_{\nu_{0}} \subset \mathcal{S}_{\nu}$. Theorem 5.4(d) now implies $\mathcal{S}_{\nu}=\mathcal{S}_{\nu}=\mathcal{S}_{\nu_{0}}$.

REMARK 5.8. By Theorems 5.7(c) and 5.4(f), $\nu^{\circ}$ satisfies condition (M) whenever $\nu$ does However, we shall soon obtain a far stronger result.

THEOREM 5.9. $\nu_{o}$ satisfies condition (M).

PROOF. By Definition 4.4, if $E \in \mathcal{S}_{\nu_{o}}$, then $\nu_{o}(X)=\nu_{o}(E)+\nu_{o}\left(E^{\prime}\right)$.

To show the converse, suppose $\nu_{o}(X)=\nu_{o}(E)+\nu_{o}\left(E^{\prime}\right)$ for some $E \subset X$. Since $\nu^{\circ}$ is finite, Theorem 5.2(c) implies $\nu_{o}\left(E^{\prime}\right)+\nu^{\circ}(E)=\nu(X)$. Therefore,

$$
\nu_{o}\left(E^{\prime}\right)+\nu^{\circ}(E)=\nu(X)=\nu_{o}(X)=\nu_{o}(E)+\nu_{o}\left(E^{\prime}\right) .
$$

All terms are finite, so $\nu^{\circ}(E)=\nu_{o}(E)$. By Theorem 5.4(c), it follows that $E \in \mathcal{S}_{\nu_{0}}$.

We now see that $E \in \mathcal{S}_{\nu_{o}}$ if and only if $\nu_{o}(X)=\nu_{o}(E)+\nu_{o}\left(E^{\prime}\right)$, so $\nu_{o}$ satisfies condition (M).

We now wish to examine the concept of regularity for finitely subadditive outer measures. To facilitate this discussion, we define a closely associated property.

DEFINITION 5.10. A finite, finitely subadditive outer measure $\nu$ on $\mathcal{P}(X)$ will be called approximately regular if $\nu=\nu^{\circ}$ on $\mathcal{P}(X)$.

The concepts of regular and approximately regular coincide in the standard theory of countably subadditive outer measures, but this need not be the case in the theory of finitely subadditive outer measures. Nevertheless, a finite, finitely subadditive outer measure which is regular will also be approximately regular. This latter concept provides us with a condition on $\nu$ weaker than full regularity but which still guarantees that $\nu$ satisfies condition (M).

THEOREM 5.11. If $\nu$ is approximately regular, then $\nu$ satisfies condition (M).

PROOF. Since it is clear that $\nu(X)=\nu(E)+\nu\left(E^{\prime}\right)$ if $E \in \mathcal{S}_{\nu}$, we need only show the converse. 
Suppose $\nu(X)=\nu(E)+\nu\left(E^{\prime}\right)$ for some $E \subset X$. If $A \in \mathcal{S}_{\nu}$, then using $E$ and $E^{\prime}$ as test sets in Definition 3.2, we have

$$
\begin{aligned}
& \nu(E)=\nu(E \cap A)+\nu\left(E \cap A^{\prime}\right), \\
& \nu\left(E^{\prime}\right)=\nu\left(E^{\prime} \cap A\right)+\nu\left(E^{\prime} \cap A^{\prime}\right) .
\end{aligned}
$$

Adding these equations and using the subadditivity of $\nu$, we obtain

$$
\begin{aligned}
\nu(X) & =\left[\nu(E \cap A)+\nu\left(E^{\prime} \cap A\right)\right]+\left[\nu\left(E \cap A^{\prime}\right)+\nu\left(E^{\prime} \cap A^{\prime}\right)\right] \\
& \geq \nu(A)+\nu\left(A^{\prime}\right)=\nu(X) .
\end{aligned}
$$

Hence,

$$
\nu(E \cap A)+\nu\left(E^{\prime} \cap A\right)+\nu\left(E \cap A^{\prime}\right)+\nu\left(E^{\prime} \cap A^{\prime}\right)=\nu(A)+\nu\left(A^{\prime}\right) .
$$

Since all quantities are finite, we can subtract the inequality $\nu\left(A^{\prime}\right) \leq \nu\left(E \cap A^{\prime}\right)+\nu\left(E^{\prime} \cap A^{\prime}\right)$ from (5.1) to obtain $\nu(A) \geq \nu(E \cap A)+\nu\left(E^{\prime} \cap A\right)$. Since $\nu$ is subadditive and $\nu=\nu^{\circ}$, we actually have

$$
\nu^{\circ}(A)=\nu^{\circ}(A \cap E)+\nu^{\circ}\left(A \cap E^{\prime}\right) \text {. }
$$

We have shown that this equality holds for all $A \in \mathcal{S}_{\nu}$, but we can readily show that it also holds for all $A \subset X$. Thus, $E \in \mathcal{S}_{\nu^{\circ}}=\mathcal{S}_{\nu}$, and the proof is complete.

In an effort to shed more light on the set function $\nu^{\circ}$, we make the following definition.

DEFINITION 5.12. If $\nu$ is a finite, finitely subadditive outer measure on $\mathcal{P}(X)$, then we can define a new set function $\nu^{\infty}$ for all $E \subset X$ by

$$
\nu^{\infty}(E)=\inf \left\{\nu^{\circ}(M) \mid E \subset M \in \mathcal{S}_{\nu}\right\} .
$$

Recalling that when $\nu$ is a finite, finitely subadditive outer measure, then $\nu^{\circ}$ is a finite, submodular outer measure and $\mathcal{S}_{\nu}$ is an algebra, we can readily apply the general theory of Sections 3 and 4 to this definition to establish the next lemma.

LEMMA 5.13. The set function $\nu^{\infty 0}$ is a finite, submodular outer measure on $\mathcal{P}(X)$ and possesses the following properties:

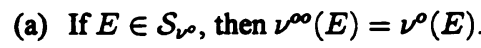

(b) $\nu^{\infty}(X)=\nu^{\circ}(X)=\nu(X)<\infty$.

(c) For every $E \subset X, \nu^{\circ}(E) \leq \nu^{\infty}(E)$.

(d) $\mathcal{S}_{\nu 00}$ is an algebra and $\nu^{\infty 0}=\left.\nu^{\infty 0}\right|_{S_{\nu 0}}$ is a finitely additive measure on $\mathcal{S}_{\nu 00}$.

This new set function reveals important new information about the outer measure $\nu^{\circ}$ and enables us to strengthen significantly the conclusions of Theorem 5.4(d,f).

THEOREM 5.14. (a) $\nu^{\circ}$ is approximately regular.

(b) $\nu^{\circ}$ satisfies condition (M).

(c) $\mathcal{S}_{\nu_{0}}=\mathcal{S}_{\nu_{0}}$.

PROOF. (a) For any $A \subset X$, Theorem 5.4(d,e) implies

$$
\begin{aligned}
\nu^{\infty}(A) & =\inf \left\{\nu^{\circ}(M) \mid A \subset M \in \mathcal{S}_{\nu^{\circ}}\right\} \\
& \leq \inf \left\{\nu(M) \mid A \subset M \in \mathcal{S}_{\nu}\right\} \\
& =\nu^{\circ}(A) .
\end{aligned}
$$

It follows immediately from Lemma $5.13(\mathrm{c})$ that $\nu^{\circ 0}(A)=\nu^{\circ}(A)$ for all $A \subset X$, so $\nu^{\circ}$ is approximately regular.

(b) Clear, by Theorem 5.11 and part (a).

(c) A direct consequence of part (b) and Theorem 5.4(f). 


\section{FURTHER EXAMPLES AND APPLICATIONS}

In this section we show the efficiency of the general theory discussed above in Sections 3, 4, and 5 when applied to some familiar lattice related set functions. We shall soon see that the nature and special properties of these set functions are revealed almost instantly. No longer will we need to examine each function individually to ascertain its nature or to labor tediously in an effort to uncover its special properties. Most of these facts will be virtually self-evident once the function is defined.

Throughout this section, $X$ will denote an arbitrary set and $\mathcal{L}$ a lattice of subsets of $X$. We shall always assume that $\emptyset, X \in \mathcal{L}$. All other notation and terminology in this section will conform to that introduced in Section 2. Beginning with the set function $\mu^{\prime}$ defined in that section we have

THEOREM 6.1. The set function $\mu^{\prime}$ as given by Definition 2.7 is a submodular outer measure possessing the following properties:

(a) $\mu^{\prime}(X)<\infty$.

(b) $\mu^{\prime}=\mu$ on $\mathcal{L}^{\prime}$.

(c) $\mu \leq \mu^{\prime}$ on $\mathcal{A}(\mathcal{L})$.

PROOF. $\mathcal{L}^{\prime}$ is a lattice, so it is a covering class which is closed under finite unions. Since $\mu \in M(\mathcal{L})$, it is finite and nonnegative on $\mathcal{L}^{\prime}$, and $\mu(\emptyset)=0$. Furthermore, $\mu$ is necessarily submodular (hence, finitely subadditive) on $\mathcal{L}^{\prime}$, and monotone. Consequently, Theorems 3.5 and 3.6 imply that $\mu^{\prime}$ is a submodular outer measure on $\mathcal{P}(X)$ such that $\mu^{\prime}(X)<\infty$ and $\mu^{\prime}=\mu$ on $\mathcal{L}^{\prime}$. Property (c) follows easily as a consequence of the definition of $\mu^{\prime}$ and the monotonicity of $\mu$.

REMARK 6.2. Obviously, $\bar{\mu}^{\prime}=\mu^{\prime}||_{\mu^{\prime}}$ is a finitely additive measure on $\mathcal{S}_{\mu^{\prime}}$, the $\mu^{\prime}$-measurable sets. In the special case for $\mu \in I(\mathcal{L}), \mu^{\prime}$ is also regular and therefore satisfies condition (M).

We turn our attention now to the familiar set function $\mu_{i}$ to see how the theory reveals not only its familiar characteristics, but also its strong connection to $\mu^{\prime}$.

DEFINITION 6.3. Let $\mathcal{L} \subset \mathcal{P}(X)$ be a lattice and $\mu \in M(\mathcal{L})$. For all $E \subset X$, we define the set function $\mu_{i}$ on $\mathcal{P}(X)$ by

$$
\mu_{i}(E)=\sup \{\mu(L) \mid L \subset E, L \in \mathcal{L}\}
$$

From this definition and Theorems 6.1 and 4.2 , we see that $\mu_{i}$ is the inner measure determined by $\mu^{\prime}$. Furthermore, Theorem 4.2 also reveals that $\mu_{i}$ is supermodular, and if $E \in \mathcal{S}_{\mu^{\prime}}$, then $\mu_{i}(E)=\mu^{\prime}(E)$. Combining this result with Theorem 4.6 and Corollary 4.7 shows that $S_{\mu^{\prime}} \subset \mathcal{S}_{\mu}=\left\{E \subset X \mid \mu_{i}(E)=\mu^{\prime}(E)\right\}$. This result opens the door to an even stronger and more interesting result, namely

THEOREM 6.4. $\mathcal{S}_{\mu^{\prime}}=\left\{E \subset X \mid \mu_{i}(E)=\mu^{\prime}(E)\right\}$.

PROOF. In view of the comments preceding this theorem, we need only show that $\mathcal{S}_{\mu_{1}} \subset \mathcal{S}_{\mu^{\prime}}$.

Let $E \in \mathcal{S}_{\mu}$. Given $\epsilon>0$, there exists an $L \in \mathcal{L}$ such that $L \subset E$ and

$$
\mu_{i}(E)<\mu(L)+\epsilon / 2 .
$$

Also, there is an $\tilde{L}^{\prime} \in \mathcal{L}^{\prime}$ such that $E \subset \tilde{L}^{\prime}$ and

$$
\mu\left(\tilde{L}^{\prime}\right)<\mu^{\prime}(E)+\epsilon / 2
$$

Combining (6.1) and (6.2) with the fact that $E \in \mathcal{S}_{\mu}$, we see that there exist $L \in \mathcal{L}$ and $\tilde{L}^{\prime} \in \mathcal{L}^{\prime}$ such that $L \subset E \subset \tilde{L}^{\prime}$ and

$$
\mu\left(\tilde{L}^{\prime}\right)<\mu^{\prime}(E)+\epsilon / 2=\mu_{i}(E)+\epsilon / 2<\mu(L)+\epsilon .
$$

To show that $E$ is $\mu^{\prime}$-measurable, let $A^{\prime} \in \mathcal{L}^{\prime}$. Then $A^{\prime} \cap E^{\prime} \subset A^{\prime} \cap L^{\prime}$, so by the monotonicity of $\mu^{\prime}$ and Theorem 6.1(b), we have

$$
\mu^{\prime}\left(A^{\prime} \cap E^{\prime}\right) \leq \mu^{\prime}\left(A^{\prime} \cap L^{\prime}\right)=\mu\left(A^{\prime} \cap L^{\prime}\right) .
$$


On the other hand, $A^{\prime} \cap E \subset A^{\prime} \cap \tilde{L}^{\prime}$, so

$$
\mu^{\prime}\left(A^{\prime} \cap E\right) \leq \mu^{\prime}\left(A^{\prime} \cap \tilde{L}^{\prime}\right)=\mu\left(A^{\prime} \cap \tilde{L}^{\prime}\right) .
$$

Now $\mu$ is modular on $\mathcal{L}^{\prime}$, so

$$
\mu\left(A^{\prime} \cap \tilde{L}^{\prime}\right)=\mu\left(A^{\prime}\right)+\mu\left(\tilde{L}^{\prime}\right)-\mu\left(A^{\prime} \cup \tilde{L}^{\prime}\right) .
$$

Combining this result with (6.3) and the monotonicity of $\mu$ yields

$$
\begin{aligned}
\mu\left(A^{\prime} \cap \tilde{L}^{\prime}\right) & \leq \mu\left(A^{\prime}\right)+\mu(L)-\mu\left(A^{\prime} \cup L\right)+\epsilon \\
& =\mu\left(A^{\prime} \cap L\right)+\epsilon,
\end{aligned}
$$

where the equality follows by modularity. It now follows from (6.4), (6.5), (6.7), Theorem 6.1(b), and the finite additivitiy of $\mu$ that

$$
\begin{aligned}
\mu^{\prime}\left(A^{\prime} \cap E\right)+\mu^{\prime}\left(A^{\prime} \cap E^{\prime}\right) & \leq \mu\left(A^{\prime} \cap \tilde{L}^{\prime}\right)+\mu\left(A^{\prime} \cap L^{\prime}\right) \\
& \leq \mu\left(A^{\prime} \cap L\right)+\mu\left(A^{\prime} \cap L^{\prime}\right)+\epsilon \\
& =\mu\left(A^{\prime}\right)+\epsilon \\
& =\mu^{\prime}\left(A^{\prime}\right)+\epsilon .
\end{aligned}
$$

Clearly this holds for all $\epsilon>0$, so

$$
\mu^{\prime}\left(A^{\prime} \cap E\right)+\mu^{\prime}\left(A^{\prime} \cap E^{\prime}\right) \leq \mu^{\prime}\left(A^{\prime}\right),
$$

for any $A^{\prime} \in \mathcal{L}^{\prime}$. It now follows easily that (6.8) holds for any $A \subset X$, and therefore $E \in \mathcal{S}_{\mu^{\prime}}$.

This leads immediately to two important results concerning $\mu^{\prime}$.

COROLLARY 6.5. If $\mu \in M(\mathcal{L})$, then $\mu^{\prime}$ satisfies condition (M).

PROOF. This follows immediately by Theorems 6.4 and 4.9 .

COMMENT. We see now that $\mu^{\prime}$ is an important example of a finitely subadditive outer measure which satisfies condition $(M)$ but is not necessarily regular.

THEOREM 6.6. If $\mu \in M(\mathcal{L})$, then

$$
\mathcal{S}_{\mu^{\prime}} \cap \mathcal{L}=\left\{L \in \mathcal{L} \mid \mu^{\prime}(L)=\mu(L)\right\}
$$

PROOF. Clear from Theorem 6.4.

REMARK 6.7. Some simple but important consequences of this theorem are: (a) $\mu \in M_{R}(\mathcal{L})$ if and only if $\mu=\mu^{\prime}$ on $\mathcal{L}$, and (b) $\mu \in M_{R}(\mathcal{L})$ if and only if $\mathcal{A}(\mathcal{L}) \subset \mathcal{S}_{\mu^{\prime}}$.

This latter result is the key that finally reveals conditions under which $\mu^{\prime}$ is regular: namely, $\mu^{\prime}$ is regular if $\mathcal{L}^{\prime}$ is $\delta$ and $\mu \in M_{R}(\mathcal{L})$.

We turn our attention now to the well known countably subadditive outer measure $\mu^{\prime \prime}$ defined earlier in Section 2. Some important facts to recall about $\mu^{\prime \prime}$ are that $\mu^{\prime \prime} \leq \mu$ on $\mathcal{L}^{\prime}, \mu \leq \mu^{\prime \prime}$ on $\mathcal{L}$, and $\mu^{\prime \prime}(X)=\mu(X)<\infty$, where $\mu \in M_{\sigma}(\mathcal{L})$. Also, $\mathcal{S}_{\mu^{\prime \prime}}$ is a $\sigma$-algebra and $\mu^{\prime \prime} \mid \mathcal{S}_{\iota^{\prime \prime}}$ is a countably additive measure. In the special case for $\mu \in I_{\sigma}(\mathcal{L}), \mu^{\prime \prime}$ is always regular.

While we require that $\mu \in M_{\sigma}(\mathcal{L})$ in order to ensure that $\mu^{\prime \prime}$ is not trivial, we can improve significantly upon the above-mentioned results for $\mu^{\prime \prime}$ by imposing a stronger condition on $\mu$, namely that $\mu \in M_{\sigma *}(\mathcal{L})$.

THEOREM 6.8. If $\mu \in M_{\sigma^{*}}(\mathcal{L})$, then $\mu^{\prime \prime}=\mu^{\prime}=\mu$ on $\mathcal{L}^{\prime}$.

PROOF. From the introductory remarks above about $\mu^{\prime \prime}$ and by Theorem 6.1, we always have

$$
\mu^{\prime \prime} \leq \mu=\mu^{\prime} \text { on } \mathcal{L}^{\prime} \text {. }
$$


To show the reverse inequality, let $L^{\prime} \in \mathcal{L}^{\prime}$, and choose any $\epsilon>0$. By the definition of $\mu^{\prime \prime}$, there exists a sequence $\left\{L_{n}\right\}_{n=1}^{\infty} \subset \mathcal{L}$ such that $L^{\prime} \subset \bigcup_{n=1}^{\infty} L_{n}^{\prime}$ and

$$
\sum_{n=1}^{\infty} \mu\left(L_{n}^{\prime}\right)<\mu^{\prime \prime}\left(L^{\prime}\right)+\epsilon .
$$

It is clear that $L^{\prime}=L^{\prime} \cap\left(\bigcup_{n=1}^{\infty} L_{n}^{\prime}\right)=\bigcup_{n=1}^{\infty}\left(L^{\prime} \cap L_{n}^{\prime}\right)$, and if $N$ is a positive integer, then

$$
\bigcup_{n=1}^{N}\left(L^{\prime} \cap L_{n}^{\prime}\right) \uparrow \bigcup_{n=1}^{\infty}\left(L^{\prime} \cap L_{n}^{\prime}\right)=L^{\prime} \in \mathcal{L}^{\prime} .
$$

The monotonicity and the finite subadditivity of $\mu$ imply that, for any $N$,

$$
\begin{aligned}
\mu\left(\bigcup_{n=1}^{N}\left(L^{\prime} \cap L_{n}^{\prime}\right)\right) & \leq \sum_{n=1}^{N} \mu\left(L^{\prime} \cap L_{n}^{\prime}\right) \leq \sum_{n=1}^{N} \mu\left(L_{n}^{\prime}\right) \\
& \leq \sum_{n=1}^{\infty} \mu\left(L_{n}^{\prime}\right)<\mu^{\prime \prime}\left(L^{\prime}\right)+\epsilon .
\end{aligned}
$$

Consequently, applying the hypothesis to (6.11) and (6.12) yields

$$
\begin{aligned}
\mu\left(L^{\prime}\right) & =\mu\left(\bigcup_{n=1}^{\infty}\left(L^{\prime} \cap L_{n}^{\prime}\right)\right)=\sup _{N} \mu\left(\bigcup_{n=1}^{N}\left(L^{\prime} \cap L_{n}^{\prime}\right)\right) \\
& \leq \mu^{\prime \prime}\left(L^{\prime}\right)+\epsilon .
\end{aligned}
$$

Since $\epsilon$ was arbitrary, this implies that $\mu\left(L^{\prime}\right) \leq \mu^{\prime \prime}\left(L^{\prime}\right)$, and consequently that $\mu \leq \mu^{\prime \prime}$ on $\mathcal{L}^{\prime}$. Combining this with (6.9) completes the proof.

An immediate consequence of this result is

COROLLARY 6.9. If $\mu \in M^{\sigma}(\mathcal{L})$, then $\mu^{\prime \prime}=\mu^{\prime}=\mu$ on $\mathcal{L}^{\prime}$.

PROOF. Clear, since $M^{\sigma}(\mathcal{L}) \subset M_{\sigma *}(\mathcal{L})$.

If $\mu \in M_{\sigma}(\mathcal{L})$ and $\mu^{\prime \prime}$ is regular, then the converse of Theorem 6.8 holds.

THEOREM 6.10. Let $\mu \in M_{\sigma}(\mathcal{L})$. If $\mu^{\prime \prime}$ is regular and $\mu^{\prime \prime}=\mu^{\prime}=\mu$ on $\mathcal{L}^{\prime}$, then $\mu \in M_{\sigma *}(\mathcal{L})$.

PROOF. Let $\left\{L_{n}^{\prime}\right\}_{n=1}^{\infty} \subset \mathcal{L}^{\prime}$ be any sequence such that $L_{n}^{\prime} \uparrow$ and $\bigcup_{n=1}^{\infty} L_{n}^{\prime} \in \mathcal{L}^{\prime}$. Let $L^{\prime}=\bigcup_{n=1}^{\infty} L_{n}^{\prime}$. Then $L_{n}^{\prime} \uparrow L^{\prime} \in \mathcal{L}^{\prime}$. By hypothesis, $\mu^{\prime \prime}$ is regular and $\mu^{\prime \prime}=\mu$ on $\mathcal{L}^{\prime}$, so it follows that

$$
\begin{aligned}
\mu\left(L^{\prime}\right) & =\mu^{\prime \prime}\left(L^{\prime}\right)=\mu^{\prime \prime}\left(\lim _{n} L_{n}^{\prime}\right)=\lim _{n} \mu^{\prime \prime}\left(L_{n}^{\prime}\right)^{\prime} \\
& =\lim _{n} \mu\left(L_{n}^{\prime}\right) .
\end{aligned}
$$

Hence,

$$
\mu\left(\bigcup_{n=1}^{\infty} L_{n}^{\prime}\right)=\mu\left(L^{\prime}\right)=\lim _{n} \mu\left(L_{n}^{\prime}\right)=\sup _{n} \mu\left(L_{n}^{\prime}\right) .
$$

This means that $\mu \in M_{\sigma *}(\mathcal{L})$.

Since $\mu^{\prime \prime}$ is always regular for $\mu \in I_{\sigma *}(\mathcal{L})$, the following corollary is an immediate consequence of Theorems 6.8 and 6.10 .

COROLLARY 6.11. If $\mu \in I_{\sigma}(\mathcal{L})$, then $\mu \in I_{\sigma *}(\mathcal{L})$ if and only if $\mu^{\prime \prime}=\mu^{\prime}=\mu$ on $\mathcal{L}^{\prime}$.

REMARK. Before we conclude our discussion of the outer measure $\mu^{\prime \prime}$, we note two additional results of interest concerning regularity for $\mu^{\prime \prime}$. First, in a spirit paralleling Theorem 6.10 , if $\mu \in M_{\sigma}(\mathcal{L})$ and $\mu^{\prime \prime}$ is regular with $\mu^{\prime \prime}=\mu$ on $\mathcal{L}$, then $\mu \in M^{o}(\mathcal{L})$. On the other hand, if $\mu \in M_{R}^{o}(\mathcal{L})$, then $\mu^{\prime \prime}$ is regular and submodular. 
To illustrate further the application of the general principles discussed in Sections 3 and 4, we now define two new set functions related to a lattice $\mathcal{L}$ and the set of measures $M(\mathcal{L})$.

DEFINITION 6.12. If $\mu \in M(\mathcal{L})$ and $\mu_{i}$ is the inner measure of Definition 6.3, then for all $E \subset X$, we define the set function $\bar{\mu}$ on $\mathcal{P}(X)$ by

$$
\bar{\mu}(E)=\inf \left\{\mu_{i}\left(L^{\prime}\right) \mid E \subset L^{\prime}, L \in \mathcal{L}\right\} .
$$

It follows immediately that $\mu_{2} \leq \bar{\mu} \leq \mu^{\prime}$ on $\mathcal{P}(X), \mu_{i}=\bar{\mu} \leq \mu^{\prime}=\mu$ on $\mathcal{L}^{\prime}$, and $\mu=\mu_{i} \leq \bar{\mu} \leq \mu^{\prime}$ on $\mathcal{L}$. If we define a $\bar{\mu}$-measurable set in the usual way, then $\mathcal{S}_{\mu}$ is an algebra and $\left.\bar{\mu}\right|_{\mathcal{S}_{\mu}}$ is a finitely additive measure. Unfortunately, because $\mu_{i}$ is an inner measure, $\bar{\mu}$ is not quite an outer measure, lacking only the property of finite subadditivity. This defect is remedied, however, if $\mathcal{L}$ is normal.

THEOREM 6.13. If $\mathcal{L}$ is a normal lattice and $\mu \in M(\mathcal{L})$, then:

(a) $\mu_{i}$ is finitely subadditive on $\mathcal{L}^{\prime}$.

(b) $\bar{\mu}$ is a finitely subadditive outer measure on $\mathcal{P}(X)$.

(c) $\mathcal{L} \subset \mathcal{A}(\mathcal{L}) \subset \mathcal{S}_{\boldsymbol{\mu}}$.

(d) $\bar{\mu}=\mu^{\prime}$ on $\mathcal{L}$.

(e) $\bar{\mu}$ is submodular.

PROOF. (a) Let $L_{1}, L_{2} \in \mathcal{L}$ and $\epsilon>0$ be given. By the definition of $\mu_{i}$, there exists a set $A \in \mathcal{L}$ such that $A \subset L_{1}^{\prime} \cup L_{2}^{\prime}$ and

$$
\mu\left(L_{1}^{\prime} \cup L_{2}^{\prime}\right)<\mu(A)+\epsilon .
$$

Since $\mathcal{L}$ is normal, there exist $A_{k} \in \mathcal{L}, k=1,2$, such that $A_{k} \subset L_{k}^{\prime}$ and $A=A_{1} \cup A_{2}$. The finite subadditivity of $\mu$ implies

$$
\mu(A) \leq \mu\left(A_{1}\right)+\mu\left(A_{2}\right) .
$$

Recalling that $\mu_{i}$ is monotone and $\mu_{i}=\mu$ on $\mathcal{L}$, it follows from (6.14) and (6.15) that

$$
\begin{aligned}
\mu_{i}\left(L_{1}^{\prime} \cup L_{2}^{\prime}\right) & <\mu_{i}\left(A_{1}\right)+\mu_{i}\left(A_{2}\right)+\epsilon \\
& \leq \mu_{i}\left(L_{1}^{\prime}\right)+\mu_{i}\left(L_{2}^{\prime}\right)+\epsilon .
\end{aligned}
$$

This holds for all $\epsilon>0$, so we have

$$
\mu_{i}\left(L_{1}^{\prime} \cup L_{2}^{\prime}\right) \leq \mu_{i}\left(L_{1}^{\prime}\right)+\mu_{i}\left(L_{2}^{\prime}\right)
$$

Finite subadditivity now follows by induction.

(b) Since $\mathcal{L}^{\prime}$ is a covering class closed under finite unions and $\mu_{i}$ is an inner measure, then part (a) above and Theorem 3.6(a) imply that $\bar{\mu}$ is a finite, finitely subadditive outer measure on $\mathcal{P}(X)$.

(c) Let $L \in \mathcal{L}$ and $A^{\prime} \in \mathcal{L}^{\prime}$, and consider any $E, F \in \mathcal{L}$ such that $E \subset A^{\prime} \cap L^{\prime}$ and $F \subset A^{\prime} \cap E^{\prime}$ From this it is clear that $F \cap E=\emptyset$ and $E \cup F \subset A^{\prime}$. Now $\bar{\mu}=\mu_{i}$ on $\mathcal{L}^{\prime}$, so by the additivity of $\mu$ on $\mathcal{L}$ and the definition of $\mu_{i}$ we have

$$
\bar{\mu}\left(A^{\prime}\right)=\mu_{i}\left(A^{\prime}\right) \geq \mu(E \cup F)=\mu(E)+\mu(F) .
$$

The choice of $F$ was arbitrary, so it follows that

$$
\begin{aligned}
\bar{\mu}\left(A^{\prime}\right) & \geq \mu(E)+\sup \left\{\mu(F) \mid F \subset A^{\prime} \cap E^{\prime}, F \in \mathcal{L}\right\} \\
& =\mu(E)+\mu_{i}\left(A^{\prime} \cap E^{\prime}\right) \\
& =\mu(E)+\bar{\mu}\left(A^{\prime} \cap E^{\prime}\right) .
\end{aligned}
$$

Clearly $L \subset E^{\prime}$, so $A^{\prime} \cap L \subset A^{\prime} \cap E^{\prime}$, and then the monotonicity of $\bar{\mu}$ implies that

$$
\bar{\mu}\left(A^{\prime}\right) \geq \mu(E)+\bar{\mu}\left(A^{\prime} \cap L\right) .
$$

Finally, since $E$ was an arbitrary choice, inequality (6.16) implies 


$$
\begin{aligned}
\bar{\mu}\left(A^{\prime}\right) & \geq \sup \left\{\mu(E) \mid E \subset A^{\prime} \cap L^{\prime}, E \in \mathcal{L}\right\}+\bar{\mu}\left(A^{\prime} \cap L\right) \\
& =\mu_{2}\left(A^{\prime} \cap L^{\prime}\right)+\bar{\mu}\left(A^{\prime} \cap L\right) \\
& =\bar{\mu}\left(A^{\prime} \cap L^{\prime}\right)+\bar{\mu}\left(A^{\prime} \cap L\right) .
\end{aligned}
$$

This statement holds for all $A^{\prime} \in \mathcal{L}^{\prime}$, but it is not hard to show that it also holds for all $A \subset X$. Consequently, $L \in \mathcal{S}_{\bar{\mu}}$, which means that $\mathcal{L} \subset \mathcal{A}(\mathcal{L}) \subset \mathcal{S}_{\bar{\mu}}$.

(d) Let $L \in \mathcal{L}$. Then by part (c), $L \in \mathcal{S}_{\mu}$, and since $\mathcal{S}_{\mu}$ is an algebra, we also have $L^{\prime} \in \mathcal{S}_{\mu}$. Since $\left.\bar{\mu}\right|_{\mathcal{S}_{\bar{\mu}}}$ is a finitely additive measure, it follows that $\bar{\mu}(X)=\bar{\mu}(L)+\bar{\mu}\left(L^{\prime}\right)$, and from Theorem 4.2, $\mu(X)=\mu_{2}\left(L^{\prime}\right)+\mu^{\prime}(L)$. Consequently,

$$
\bar{\mu}(L)+\bar{\mu}\left(L^{\prime}\right)=\mu_{i}\left(L^{\prime}\right)+\mu^{\prime}(L) .
$$

Since $\bar{\mu}=\mu_{i}$ on $\mathcal{L}^{\prime}$ and all quantities are finite, we conclude that $\bar{\mu}(L)=\mu^{\prime}(L)$. Thus, $\bar{\mu}=\mu^{\prime}$ on $\mathcal{L}$.

(e) By part (c), we have $\mathcal{L}^{\prime} \subset \mathcal{S}_{\bar{\mu}}$, therefore $\left.\bar{\mu}\right|_{\mathcal{S}_{\bar{\mu}}}$ satisfies the modular law on $\mathcal{L}^{\prime}$. From the remarks preceding Theorem $6.13, \bar{\mu}=\mu_{i}$ on $\mathcal{L}^{\prime}$, so $\mu_{i}$ is also modular and, hence, submodular on $\mathcal{L}^{\prime}$. Theorem 3.6(b) now implies that $\bar{\mu}$ is submodular on $\mathcal{P}(X)$.

The second new set function we wish to define and examine in the light of the general principles set forth in Sections 3 and 4 is the set function $\mu_{j}$, which has a strong relationship to $\bar{\mu}$.

DEFINITION 6.14. If $\mu \in M(\mathcal{L})$ and $\mu^{\prime}$ is the outer measure of Definition 2.7, then for all $E \subset X$, we define the set function $\mu_{j}$ on $\mathcal{P}(X)$ by

$$
\mu_{j}(E)=\sup \left\{\mu^{\prime}(L) \mid E \supset L \in \mathcal{L}\right\} .
$$

From this we can readily show that if $\mathcal{L}$ is a lattice and $\mu \in M(\mathcal{L})$, then for all $E \subset X$, $\mu_{j}(E)+\bar{\mu}\left(E^{\prime}\right)=\mu(X)$. Furthermore, $\mu_{j}$ is finite, nonnegative, monotone, $\mu_{j}(\emptyset)=0$, and $\mu_{j}(X)=\mu(X)$. We shall define a $\mu_{j}$-measurable set in the standard manner and denote the class of all $\mu_{j}$-measurable sets by $\mathcal{S}_{\mu}$. As one might expect, Theorem 3.3 assures us that $\mathcal{S}_{\mu,}$ is an algebra and that $\mu_{j} \mid \mathcal{S}_{\mu}$ is a finitely additive measure. The relationship of $\mu$, to the other set functions we have discussed is summarized in

THEOREM 6.15. If $\mu \in M(\mathcal{L})$, then:

(a) $\mu_{i} \leq \mu_{j} \leq \mu^{\prime}$ on $\mathcal{P}(X)$.

(b) $\mu=\mu_{i} \leq \bar{\mu} \leq \mu_{j}=\mu^{\prime}$ on $\mathcal{L}$.

(c) $\mu_{i}=\bar{\mu} \leq \mu_{j} \leq \mu^{\prime}=\mu$ on $\mathcal{L}^{\prime}$.

(d) If $E \in \mathcal{S}_{\mu}$, then $\mu_{j}(E)=\bar{\mu}(E)$.

The proofs of these statements are straightforward and will be omitted here.

Just as $\bar{\mu}$ fails to be an outer measure unless $\mathcal{L}$ is normal, so $\mu_{j}$ similarly fails to be an inner measure without this same additional condition on $\mathcal{L}$. However, if $\mathcal{L}$ is normal, then not only does $\mu_{j}$ become countably superadditive and, hence, an inner measure, but other useful information is revealed also.

THEOREM 6.16. If $\mathcal{L}$ is a normal lattice and $\mu \in M(\mathcal{L})$, then:

(a) $\mu_{j}$ is a supermodular inner measure on $\mathcal{P}(X)$.

(b) $\mathcal{S}_{\mu_{j}}=\left\{E \subset X \mid \mu_{j}(E)=\bar{\mu}(E)\right\}$.

(c) $\mathcal{L} \subset \mathcal{A}(\mathcal{L}) \subset \mathcal{S}_{\mu} \subset \mathcal{S}_{\mu}$.

PROOF. (a) By Theorem 6.13, $\bar{\mu}$ is a submodular outer measure, so from the observations following Definition 6.14 and the finiteness of $\bar{\mu}$, it follows that $\mu_{j}(E)=\mu(X)-\bar{\mu}\left(E^{\prime}\right)=\bar{\mu}(X)-\bar{\mu}\left(E^{\prime}\right)$, for all $E \subset X$. Theorem 4.2 now implies that $\mu_{j}$ is an inner measure, and since it is not hard to show that $\mu_{j}$ is also supermodular, the proof is complete.

(b) Immediate by Theorem 4.6.

(c) A consequence of Theorem 6.13 and Corollary 4.7.

In addition to these results, we can also improve upon the conclusion of Theorem 6.15 if $\mathcal{L}$ is normal. 
THEOREM 6.17. If $\mathcal{L}$ is a normal lattice and $\mu \in M(\mathcal{L})$, then:

(a) $\mu_{i} \leq \mu_{j} \leq \bar{\mu} \leq \mu^{\prime}$ on $\mathcal{P}(X)$.

(b) $\mu=\mu_{i} \leq \mu_{j}=\bar{\mu}=\mu^{\prime}$ on $\mathcal{L}$.

(c) $\mu_{i}=\mu_{j}=\bar{\mu} \leq \mu^{\prime}=\mu$ on $\mathcal{L}^{\prime}$.

As in Theorem 6.15, the proofs of these statements are straightforward and will be omitted.

Because weakly regular measures are defined in terms of the values of the outer measure $\mu^{\prime}$ on the lattice $\mathcal{L}$, where $\mu \in M(\mathcal{L})$ (see Section 2), it is now possible to simplify the study of these measures by treating them as a natural application of the set function $\mu_{j}$. For example, we have

THEOREM 6.18. If $\mathcal{L}$ is a lattice and $\mu \in M(\mathcal{L})$, then $\mu \in M_{W}(\mathcal{L})$ if and only if $\mu_{j}=\mu$ on $\mathcal{L}^{\prime}$.

PROOF. If $\mu \in M_{W}(\mathcal{L})$, then by the definition of $\mu_{j}$, we have for all $L^{\prime} \in \mathcal{L}^{\prime}$,

$$
\mu_{j}\left(L^{\prime}\right)=\sup \left\{\mu^{\prime}(\tilde{L}) \mid L^{\prime} \supset \tilde{L} \in \mathcal{L}\right\}=\mu\left(L^{\prime}\right)
$$

Hence, $\mu_{j}=\mu$ on $\mathcal{L}^{\prime}$.

Conversely, if $\mu_{j}=\mu$ on $\mathcal{L}^{\prime}$, then it follows that for all $L^{\prime} \in \mathcal{L}^{\prime}$,

$$
\mu\left(L^{\prime}\right)=\mu_{j}\left(L^{\prime}\right)=\sup \left\{\mu^{\prime}(\tilde{L}) \mid L^{\prime} \supset \tilde{L} \in \mathcal{L}\right\} .
$$

This implies that $\mu \in M_{W}(\mathcal{L})$.

Using this theorem, it follows easily that $M_{R}(\mathcal{L}) \subset M_{W}(\mathcal{L})$, but we can strengthen this result if $\mathcal{L}$ is normal.

THEOREM 6.19. If $\mathcal{L}$ is a normal lattice, then $M_{R}(\mathcal{L})=M_{W}(\mathcal{L})$.

PROOF. It will suffice to show that $M_{W}(\mathcal{L}) \subset M_{R}(\mathcal{L})$, so let $\mu \in M_{W}(\mathcal{L})$ and $L^{\prime} \in \mathcal{L}^{\prime}$. Clearly,

$$
\mu_{j}\left(L^{\prime}\right)+\bar{\mu}(L)=\mu(X)=\mu\left(L^{\prime}\right)+\mu(L),
$$

so by Theorem 6.18 and the finiteness of all terms, we deduce that $\bar{\mu}(L)=\mu(L)$. Hence, $\bar{\mu}=\mu$ on $\mathcal{L}$. Since $\mathcal{L}$ is normal, Theorem 6.17(b) implies that $\bar{\mu}=\mu^{\prime}$ on $\mathcal{L}$, so $\mu=\mu^{\prime}$ on $\mathcal{L}$. Recalling Remark 6.7(a), we see that $\mu \in M_{R}(\mathcal{L})$.

As a final illustration of how our general principles may be applied to specific set functions in order to simplify their study, we apply the results of Section 5 to the well known set functions $\mu^{\prime}$ and $\mu_{2}$ discussed earlier. We shall see that this application also reveals new and unexpected information about the set functions $\bar{\mu}$ and $\mu_{j}$.

Recall from our earlier results that $\mu^{\prime}$ is a finite, submodular outer measure which satisfies condition $(M), \mathcal{S}_{\mu^{\prime}}$ is an algebra, and $\mathcal{S}_{\mu^{\prime}}=\mathcal{S}_{\mu^{\prime}}=\left\{E \subset X \mid \mu_{\imath}(E)=\mu^{\prime}(E)\right\}$. Letting $\nu=\mu^{\prime}$ in Definition 5.1, we have for all $E \subset X$,

$$
\begin{aligned}
& \nu^{\circ}(E)=\inf \left\{\mu^{\prime}(M) \mid E \subset M \in \mathcal{S}_{\mu^{\prime}}\right\} \\
& \nu_{o}(E)=\sup \left\{\mu^{\prime}(M) \mid E \supset M \in \mathcal{S}_{\mu^{\prime}}\right\}
\end{aligned}
$$

The following results are immediate consequences of the general principles derived in Section 5.

PROPOSITION 6.20. (a) $\nu^{\circ}$ is a finite, submodular outer measure.

(b) $\nu_{o}$ is a finite, supermodular inner measure such that $\nu_{o}(E)=\mu^{\prime}(X)-\nu^{\circ}\left(E^{\prime}\right)$, for all $E \subset X$.

(c) For all $E \subset X, \nu_{o}(E) \leq \mu_{i}(E) \leq \mu^{\prime}(E) \leq \nu^{\circ}(E)$.

PROOF. (a) and (b) are obvious consequences of Theorem 5.2(b,c). To prove part (c), we note that by Theorems 5.2(a) and 4.2(d), if $E \subset X$, then $\nu_{o}(E) \leq \mu^{\prime}(E) \leq \nu^{\circ}(E)$ and $\mu_{i}(E) \leq \mu^{\prime}(E) \leq \nu^{\circ}(E)$. It remains only to show that $\nu_{o}(E) \leq \mu_{i}(E)$.

Consider any $M \in \mathcal{S}_{\mu^{\prime}}$ such that $M \subset E$. By Theorem 6.4 and the monotonicity of $\mu_{2}$, $\mu^{\prime}(M)=\mu_{i}(M) \leq \mu_{i}(E)$. Hence,

$$
\nu_{o}(E)=\sup \left\{\mu^{\prime}(M) \mid E \supset M \in \mathcal{S}_{\mu^{\prime}}\right\} \leq \mu_{i}(E) .
$$

Because $\mu^{\prime}$ satisfies condition $(M)$, we also have 
PROPOSITION 6.21. (a) If $E \in \mathcal{S}_{\mu^{\prime}}$, then $\nu_{o}(E)=\mu_{\imath}(E)=\mu^{\prime}(E)=\nu^{\circ}(E)$.

(b) If $E \subset X$ and either $\mu_{i}(E)=\mu^{\prime}(E)$ or $\nu_{o}(E)=\nu^{\circ}(E)$, then $E \in \mathcal{S}_{\mu^{\prime}}$ and $\nu_{o}(E)=\mu_{i}(E)=\mu^{\prime}(E)=\nu^{\circ}(E)$.

PROOF. Because $\mu^{\prime}$ satisfies condition $(M)$, Theorems 5.7(c) and 6.4 imply that $\mathcal{S}_{\nu_{0}}=\mathcal{S}_{\mu_{\mathrm{s}}}=\mathcal{S}_{\mu^{\prime}}=\mathcal{S}_{\nu^{\circ}}$. In particular, this means that

$$
\left\{E \subset X \mid \mu_{\imath}(E)=\mu^{\prime}(E)\right\}=\left\{E \subset X \mid \nu_{o}(E)=\nu^{\circ}(E)\right\},
$$

so that $\mu_{i}(E)=\mu^{\prime}(E)$ if and only if $\nu_{o}(E)=\nu^{\circ}(E)$.

Clearly then, if $E \in \mathcal{S}_{\mu^{\prime}}$, both equalities hold and by Proposition 6.20, $\nu_{o}(E)=\mu_{i}(E)=$ $\mu^{\prime}(E)=\nu^{\circ}(E)$. Conversely, if $\mu_{\imath}(E)=\mu^{\prime}(E)$, then $E \in \mathcal{S}_{\mu^{\prime}}$, so by the above argument, $\nu_{o}(E)=\mu_{i}(E)=\mu^{\prime}(E)=\nu^{\circ}(E)$. Similarly, if $\nu_{o}(E)=\nu^{\circ}(E)$, then by Theorem 5.7(b), $E \in \mathcal{S}_{\mu^{\prime}}$, and then the same equalities hold.

NOTE. Similar conclusions hold for any $E$ in $\mathcal{S}_{\nu}, \mathcal{S}_{\nu_{0}}$, or $\mathcal{S}_{\mu_{1}}$, since these collections coincide. Also, we note that in addition to the properties discussed above, it follows from Theorems 5.9 and 5.14 that $\nu^{\circ}$ is approximately regular and that both $\nu_{o}$ and $\nu^{\circ}$ satisfy condition $(M)$.

Because $\mathcal{S}_{\mu^{\prime}}=\mathcal{S}_{\mu_{2}}$, we can give alternate definitions for $\nu^{\circ}$ and $\nu_{0}$ when $\nu=\mu^{\prime}$ :

$$
\begin{aligned}
& \nu^{\circ}(E)=\inf \left\{\mu_{i}(M) \mid E \subset M \in \mathcal{S}_{\mu}\right\} \\
& \nu_{o}(E)=\sup \left\{\mu_{i}(M) \mid E \supset M \in \mathcal{S}_{\mu_{i}}\right\}
\end{aligned}
$$

These alternative definitions lead to some unexpected results about $\bar{\mu}$ and $\mu_{j}$ : namely, if $\mu \in M_{R}(\mathcal{L})$, then $\bar{\mu}=\mu^{\prime}=\nu^{\circ}$ and $\mu_{j}=\mu_{i}=\nu_{o}$. Consequently, when $\mu \in M_{R}(\mathcal{L})$, then $\bar{\mu}$ is a finitely subadditive outer measure and $\mu_{j}$ is an inner measure, even if $\mathcal{L}$ is not normal.

ACKNOWLEDGEMENT. The author wishes to thank the Research Released Time Committee and the Trustees of Long Island University for their generous partial support of this research work through a grant of released time from teaching duties.

\section{REFERENCES}

[1] ALEXANDROFF, A.D., Additive set functions in abstract spaces, Mat. Sb. (N.S.) 8, 50 (1940), 307-348.

[2] BACHMAN, G. and SULTAN, A., On regular extensions of measures, Pacific J. Math. 86 (1980), no. 2, 389-395.

[3] GRASSI, P., Outer measures and associated lattice properties, Internat. J. Math. \& Math. Sci., 16 (1993), no. 4, 687-694.

[4] HAHN, H. and ROSENTHAL, A., Set Functions, Univ. of New Mexico Press, Albuquerque, 1948.

[5] KNIGHT, J.E., On finitely subadditive outer measures, Jour. of Math. Sc. 7 (1996), no. 2, 91-102.

[6] MUNROE, M.E., Introduction to Measure and Integration, Addison-Wesley, Reading, Mass., 1953.

[7] PONNLEY, J., Outer measures, measurability, and lattice regular measures, Internat. J. Math. \& Math. Sci., 19 (1996), no. 2, 343-350.

[8] SIEGEL, D., Outer measures and weak regularity of measures, Internat. J. Math. \& Math. Sci., 18 (1995), no. 1, 49-58.

[9] SZETO, M., On normal lattices and separation properties of lattices, J. Indian Math. Soc., 58 (1992), no. 1, 51-64.

[10] TRAINA, C., Outer measures associated with lattice measures and their applications, Internat. $J$. Math. \& Math. Sci., 18 (1995), no. 4, 725-734.

[11] VLAD, C., Lattice separation and properties of Wallman type spaces, Ann. Mat. Pura Appl., 155 (1991), no. 4, 65-79.

[12] WALLMAN, H., Lattices and topological spaces, Ann. of Math., 39 (1938), 112-126. 


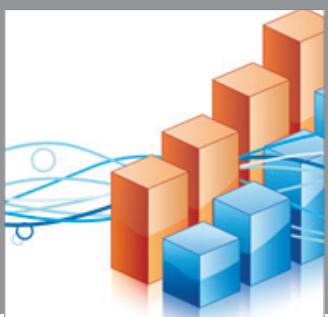

Advances in

Operations Research

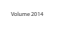

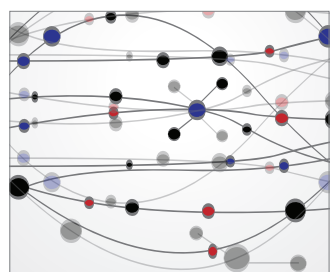

\section{The Scientific} World Journal
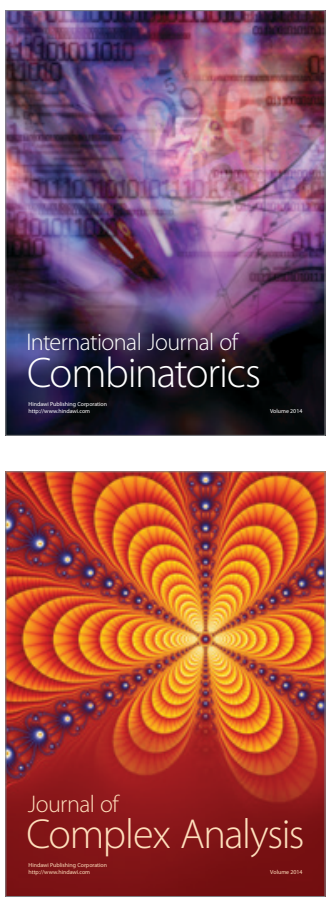

International Journal of

Mathematics and

Mathematical

Sciences
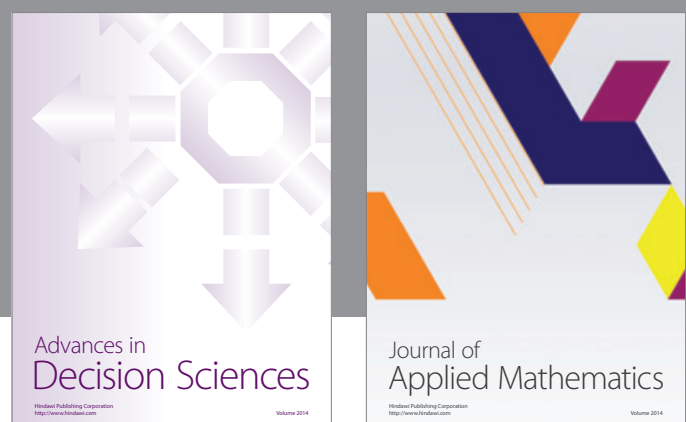

Journal of

Applied Mathematics
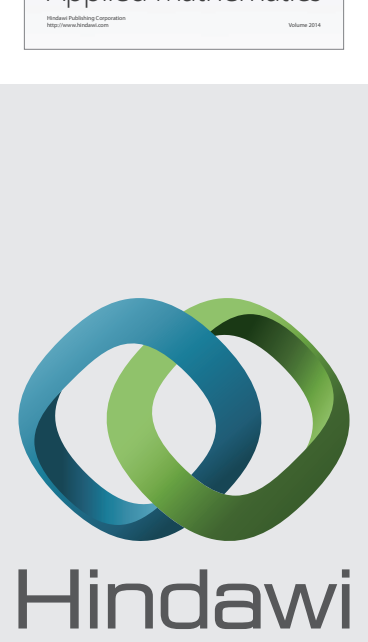

Submit your manuscripts at http://www.hindawi.com
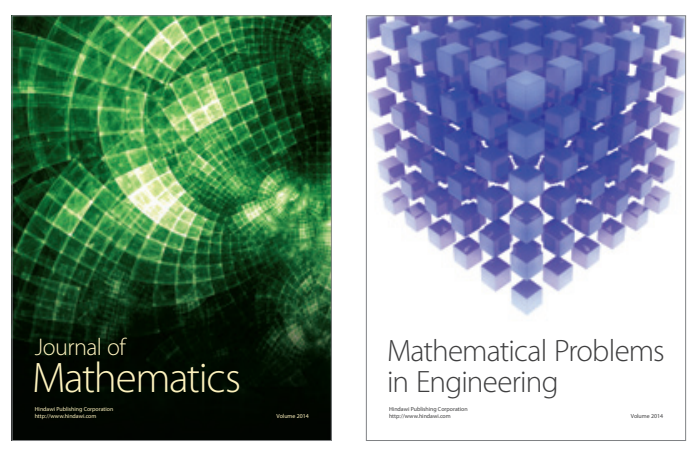

Mathematical Problems in Engineering
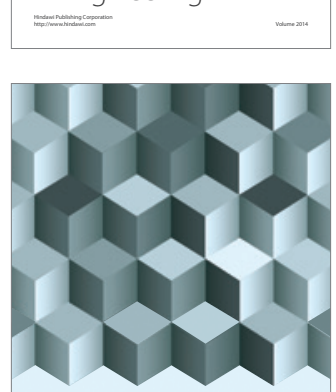

Journal of

Function Spaces
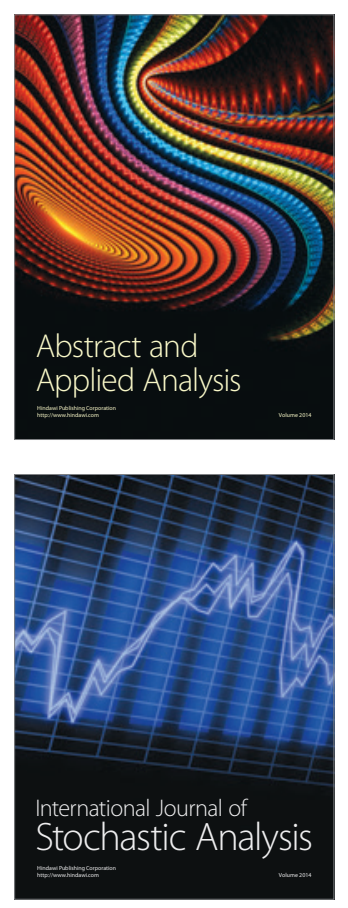

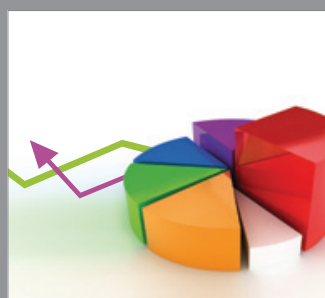

ournal of

Probability and Statistics

Promensencen
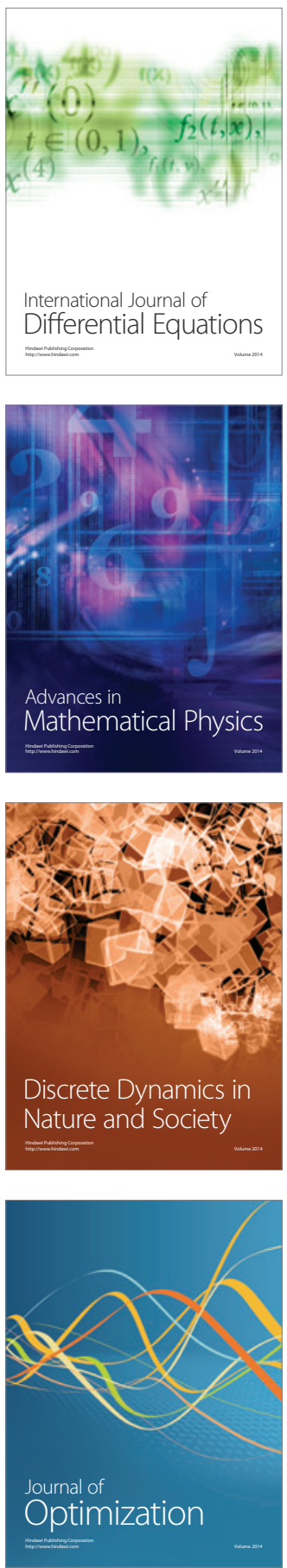